\title{
Ultracomfortable Hierarchical Nanonetwork for Highly Sensitive Pressure Sensor
}

\author{
Xin Li, ${ }^{\dagger, \S,}$, You Jun Fan, ${ }^{\dagger,,, \bullet}$ Hua Yang Li, $\stackrel{\dagger}{\dagger}$ Jin Wei Cao, ${ }^{\ddagger}$ Yu Chuan Xiao, ${ }^{\S, \perp}$ \\ Ying Wang, ${ }^{\dagger, \S}$ Fei Liang, ${ }^{\dagger, \S}$ Hai Lu Wang, ${ }^{\dagger,} \S$ Yang Jiang, ${ }^{\dagger}, \S$ Zhong Lin Wang, ${ }^{\dagger}, \S, \#$ \\ Guang Zhu,
}

${ }^{\dagger}$ CAS Center for Excellence in Nanoscience, Beijing Key Laboratory of Micro-Nano Energy and Sensor, Beijing Institute of Nanoenergy and Nanosystems, Chinese Academy of Sciences, Beijing 100083, China.

$\$$ Department of Mechanical, Materials and Manufacturing Engineering, The University of Nottingham Ningbo China, Ningbo 315100, China.

$\S$ School of Nanoscience and Technology, University of Chinese Academy of Sciences, Beijing 100049, China.

" School of Materials Science and Engineering, State Key Lab of New Ceramics and Fine Processing, Tsinghua University, Beijing 100084, China.

${ }^{\perp}$ CAS Key Laboratory of Standardization and Measurement for Nanotechnology, CAS Center for Excellence in Nanoscience, National Center for Nanoscience and Technology (NCNST), No.11 ZhongguancunBeiyitiao, Beijing 100190, China.

\# School of Materials Science and Engineering, Georgia Institute of Technology, Atlanta, GA 30332, USA.

*E-mail: zhuguang@binn.cas.cn

KEYWORDS: skin sensor, flexible electronic, silver nanowires, graphene, piezoresistive effect, hierarchical nano-network 


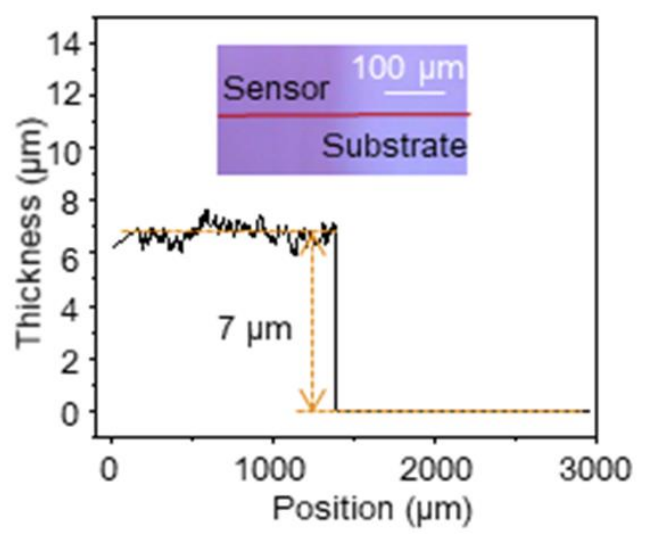

Figure S1. Thickness of the Ag NWs/GR/PANF Pressure Sensor. Height profile measured by scans along the red line of inset measuring photopragh. 
(a)

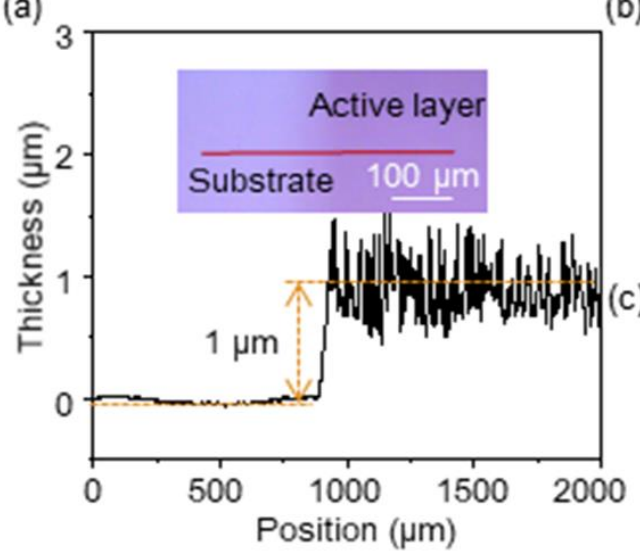

(b)

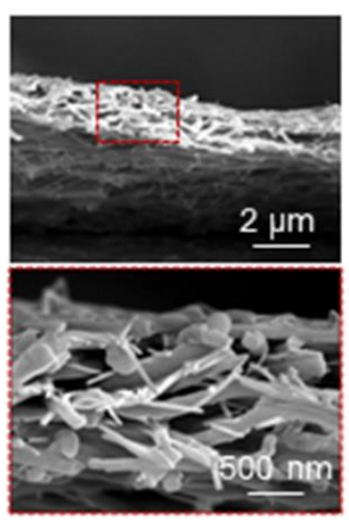

Figure S2. (a) Thickness of the Ag NWs/GR/PANF film. Height profile measured by scans along the red line of inset measuring photopragh. (b) Cross-sectional SEM images and (c) magnified views on the hierarchical Ag NWs/GR/PANF film, respectively. 


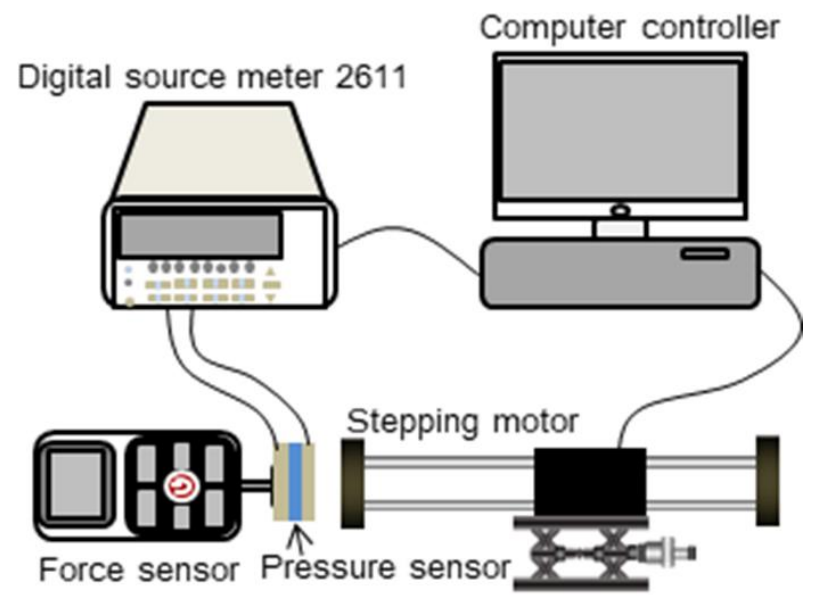

Figure S3. Schematic of the pressure sensing measurement. 
(a)

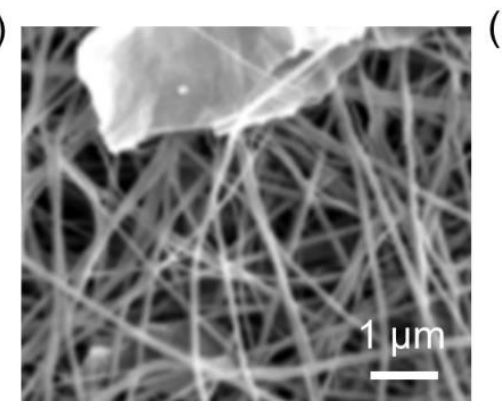

(b)

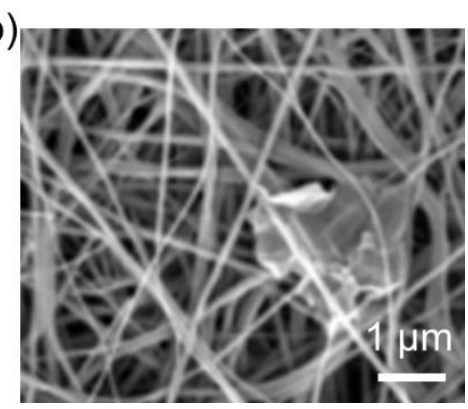

Figure S4. (a) The morphology of the hierarchical nano-network material at original state. (b) The morphology of hierarchical nano-network material after loading and unloading tests. 
Table S1. The thickness comparison among skin sensors

\begin{tabular}{|c|c|c|c|c|}
\hline $\begin{array}{l}\text { Sensor } \\
\text { Principles }\end{array}$ & Sensitivity & Materials of the sensor & Thickness & Ref. \\
\hline Piezoresistivity & $\begin{array}{c}15 \mathrm{kPa}^{-1} \text { under } \\
\text { low pressure } \\
(<100 \mathrm{~Pa})\end{array}$ & PDMS film + nanogold & $>2 \mathrm{~mm}$ & 1 \\
\hline Piezoresistivity & $10.74 \mathrm{kPa}^{-1}$ & $\begin{array}{l}\text { Conductive carbon / silicone } \\
\text { composites }\end{array}$ & $\approx 0.5 \mathrm{~mm}$ & 2 \\
\hline Capacitance & $5 \mathrm{nF} \mathrm{kPa}^{-1}$ & Home-made ionic films & $75 \mu \mathrm{m}$ & 3 \\
\hline Triboelectricity & $0.947 \mu \mathrm{A} \mathrm{kPa}^{-1}$ & $\begin{array}{l}\text { Fluorinated ethylene propylene } \\
\text { (FEP) +ITO + polypropylene (PP) }\end{array}$ & $90 \mu \mathrm{m}$ & 4 \\
\hline Piezoresistivity & $\begin{array}{c}134 \mathrm{kPa}^{-1} \text { under } \\
1.5 \mathrm{kPa}\end{array}$ & $\begin{array}{l}\text { Silver nanofibers / graphene / } \\
\text { polyamide nanofibers }\end{array}$ & $7 \mu \mathrm{m}$ & Our work \\
\hline
\end{tabular}

\section{REFERENCES:}

1. Zhang, Y.; Hu, Y.; Zhu, P.; Han, F.; Zhu, Y.; Sun, R.; Wong, C. P. Flexible and Highly Sensitive Pressure Sensor Based on Microdome-Patterned PDMS Forming with Assistance of Colloid Self-Assembly and Replica Technique for Wearable Electronics. ACS Appl. Mater. Interfaces 2017, 9, 35968-35976.

2. Huang, Y.; Chen, Y.; Fan, X.; Luo, N.; Zhou, S.; Chen, S. C.; Zhao, N.; Wong, C. P. Wood Derived Composites for High Sensitivity and Wide Linear-Range Pressure Sensing. Small 2018, 14, 1801520.

3. Zhu, Z.; Li, R.; Pan, T. Imperceptible Epidermal-Iontronic Interface for Wearable Sensing. Adv. Mater. 2018, 30, 1705122.

4. Chen, S. W.; Cao, X.; Wang, N.; Ma, L.; Zhu, H. R.; Willander, M.; Jie, Y.; Wang, Z. L. An Ultrathin Flexible Single-Electrode Triboelectric-Nanogenerator for Mechanical Energy Harvesting and Instantaneous Force Sensing. Adv. Energy Mater. 2017, 7, 1601255. 